\title{
Asthma and Gastroesophageal Reflux Disease in Children - Completing the Puzzle
}

Fernando Maria de Benedictis, ${ }^{1}$ Anna Maria Tocco ${ }^{2}$ and Giuliano Lombardi ${ }^{2}$

1. Salesi Children Hospital Foundation, Ancona, Italy; 2. Division of Pediatrics, Ospedale Santo Spirito, Pescara, Italy

$\mathrm{M}$ any findings support a possible association between gastroesophageal reflux disease (GERD) and asthma in children, but there is not enough evidence to support the causality of this association. Longitudinal studies with long-term follow-up are urgently required to cover the many gaps that persist in this area. Treatment of GERD with proton pump inhibitors (PPIS) in children with uncontrolled asthma does not substantively improve asthma outcomes but large, controlled trials in children symptomatic of both asthma and GERD are lacking. Since there are significant safety concerns for long-term PPIs use in children, physicians should carefully balance their therapeutic decisions in individual cases.

DOl: https://doi.org/10.17925/ERPD.2017.03.01.29

\section{Keywords}

Asthma, gastroesophageal reflux, comorbidity, microaspiration, $\mathrm{pH}$ measurement, proton pump inhibitors

Disclosure: Fernando Maria de Benedictis, Anna Maria Tocco and Giuliano Lombardi have no conflicts of interest to declare in relation to this article. No funding was received for the publication of this article.

Compliance with Ethics: This study involves a review of the literature and did not involve any studies with human or animal subjects performed by any of the authors.

Authorship: All named authors meet the International Committee of Medical Journal Editors (ICMJE) criteria for authorship of this manuscript, take responsibility for the integrity of the work as a whole, and have

open Access: This article is published under the Creative Commons Attribution Noncommercial License which permits any non-commercial use, distribution adaptation and reproduction provided the original author(s) and source are given appropriate credit. Received: 17 October 2016

Accepted: 25 January 2017

Citation: European Respiratory \& Pulmonary Diseases, 2017:3(1):29-31

Corresponding Author: Fernando Maria de Benedictis, Salesi Children's Hospital Foundation, 11, via Corridoni I-60123 Ancona, Italy. E: pediatria@fmdebenedictis.it
Gastroesophageal reflux (GER), the intermittent ascent of acid contents into the oesophagus, is a normal physiological process. In contrast, gastroesophageal reflux disease (GERD) is present when the reflux of acid contents causes troublesome symptoms and/or complications. ${ }^{1}$ Asthma and symptomatic GER are both common disorders in childhood and symptoms of GER are frequently reported among children with asthma. ${ }^{2.3}$ Historically, respiratory manifestations have been recognized as a potential consequence of GERD, and the relationship between asthma and GERD has been largely debated in the literature.

The aim of this article is to critically review the nature and the clinical aspects of this association in childhood and shed light on one of the most controversial fields of respiratory medicine.

\section{Exploring the association between asthma and gastroesophageal reflux disease}

Over the last 30 years, several studies have evaluated the prevalence of GERD in children with asthma. Data are controversial, the differences in the reported prevalence being determined mainly by the criteria used for definition of GERD (that is, symptoms versus laboratory investigation), the asthmatic population included (mild versus severe asthma), the design of the study (interventional, cross-over, retrospective) and the presence of a control group. It is, therefore, not surprising that the mean prevalence of GERD in children with asthma varied from $19.6 \%$ to $62.9 \%$ when GERD was identified by gastrointestinal symptoms or oesophageal pH monitoring, respectively. ${ }^{4}$ Few studies included a control group, and the prevalence of GERD was $4.8 \%$ in healthy children. The pooled odds ratio for the association between GERD and asthma was 5.6 in controlled studies. ${ }^{4}$

Exploring the relationship between asthma and GERD in children is hampered by many shortcomings. A global, evidence-based consensus on the definition of GERD in the paediatric population suggests that GERD be defined by using a "patient-centred symptom-based" method. ${ }^{5}$ However, symptom-based criteria for GERD have significant limitations in children, because reporting of symptoms may be unreliable until the age of eight years. ${ }^{5}$ Furthermore, asthma and GERD may have similar symptoms in childhood, such as nocturnal cough, chest tightness and exercise-induced discomfort, thus making difficult to determine which children actually have GERD. ${ }^{6}$ Children and adolescents with GERD more commonly manifest cough and other respiratory symptoms than the typical oesophageal complaint of heartburn in adults. ${ }^{7.8}$ Indeed, in a recent study in children with poorly controlled asthma, no gastrointestinal symptom differentiated those with and without GER identified by oesophageal pH monitoring. ${ }^{9}$ Taken together, these findings emphasise that the prevalence of GERD in asthmatic children identified in studies that used symptom scores as inclusion criteria and/or endpoints is likely biased by the inability to differentiate symptoms of asthma from those of GER. 
The majority of studies that examined GERD in children with asthma reported oesophageal pH measurement. However, these studies often selected a reflux-index threshold that was lower than that required by evidence-based guidelines, which possibly led to an overestimation of the prevalence of reflux in patients with asthma. On the other hand, pH-metry is limited by the inherent inability to detect non-acid reflux.

From a clinical point of view, the temporal interdependence between GERD and asthma is an important proof of concept for a causal relationship. This aspect was investigated in three cross-sectional studies that assessed whether respiratory symptoms (wheezing, cough) followed episodes of GERD. 10,1,1,12 These studies failed to show a consistent relationship between respiratory symptoms and periods of abnormal oesophageal pH or reflux symptoms, thus underlying that the association of GERD and asthma cannot necessarily be causal, but is rather due to chance alone.

\section{Pathophysiological mechanisms}

The pathophysiological relationship between asthma and GER has gained much attention by pulmonologists, gastroenterologists and physiologists over the years. The available evidence does not yet clearly indicate whether GER precedes and/or triggers asthma, or asthma precedes and/ or triggers GER. It is, however, likely that each one of these conditions may act as aggravating cofactor of the other.

There are several anatomic and physiological reasons that can justify the increased prevalence of GERD in asthmatic patients. Asthma is known to promote GER by different mechanisms including:

- airway obstruction with concomitant increase of negative intrathoracic pressure, that increases the pressure gradient across the lower oesophageal sphincter;

- cough-induced increase in intra-abdominal pressure, that in turn augments the gastroesophageal pressure gradient;

- lung hyperinflation, that may alter the anatomic connection of the gastroesophageal junction through the crural diaphragm; and

- asthma medications (such as beta2-agonists, aminophylline) that may induce relaxation of the lower oesophageal sphincter. ${ }^{13}$

On the other hand, two major pathophysiological mechanisms, which are not mutually exclusive, have been proposed to explain how GER may exacerbate asthma:

- microaspiration of gastric content can cause tissue injury of the airway and local inflammation (aspiration theory); and/or

- stimulation of pH-sensitive irritant receptors in the distal oesophagus may both elicit bronchospasm and increase airway hyperresponsiveness through cholinergic pathways (reflex theory).

In addition, neuroinflammatory reflexes may be involved through the release of tachykinins and other neurotransmitters in the airway by nociceptive afferent nerves, thus resulting in airway oedema, mucus secretion, vasodilation and bronchial constriction. ${ }^{14}$

Patients with GER have significantly reduced laryngopharyngeal sensitivity because of repeated exposure of mucosa to small amounts of acid, thus increasing the risk for aspiration. Chronic microaspiration of refluxate into the airway may present with chronic cough, wheeze and recurrent episodes of pneumonia, and may result in progressive lung disease. However, there is no gold standard test for diagnosing chronic lung aspiration, and determining whether aspiration is a significant cause of respiratory disease remains a challenge in paediatric medicine..$^{15}$

\section{The role of gastroesophageal reflux in children with uncontrolled asthma}

Much of the confusion around the role of GERD in asthma is likely due to the high prevalence of these two conditions in the general population. 16,17 In their clinical approach, physicians should keep in mind that asthma and GER may coexist independently. The respect of this principle may avoid misinterpretation in the evaluation process and inappropriate therapeutic decisions as well.

GERD is a recognised comorbidity in asthma, and untreated GERD has been postulated to be the cause of inadequate asthma control despite intensive anti-asthma treatment. ${ }^{18}$ In the past, some observational studies supported a treatment-response relationship for GERD in asthmatic patients. Many physicians improperly interpreted these anecdotal results as a safeconduct to prescribe anti-GER medications in patients with uncontrolled asthma and positive pH study. Indeed, these studies suffer from several design flaws and subsequent placebo-controlled trials revealed new findings. A systematic review reported that GERD treatment in patients with uncontrolled asthma (but who were not recruited specifically on the basis of reflux-associated respiratory symptoms) did not improve asthma symptoms, lung function or the use of asthma medications. ${ }^{19}$

In adults, proton pump inhibitors (PPIs) may be helpful in some patients with poorly controlled asthma who manifest reflux symptoms, but are not effective for those with asymptomatic GER. ${ }^{20,21}$ The response of asthmatic children to anti-GER treatment seems to be different from adults. The first placebo-controlled trial of omeprazole in 38 children with uncontrolled asthma and symptomatic GER showed no significant effect of omeprazole on asthma outcomes.22 In a recent double-blind, placebo-controlled study among 306 school-age children with poorly controlled asthma, the addition of lansoprazole to anti-asthma medications for 24 weeks did not improve respiratory symptoms nor lung function, but was associated with increased adverse events; the results on asthma outcomes were similar in the subgroup with a positive pH study ('silent' GER). ${ }^{9}$

Current evidence therefore does not support the routine use of antiGER medications in the treatment of poorly controlled asthma. Large controlled trials in children symptomatic of both GERD and asthma have not been conducted, and in this case the benefits of treatment, although unproven, might outweigh the risks. Other subgroups of patients, such as those with a demonstrated temporal interdependence between gastrointestinal and respiratory symptoms, may gain benefit, but proper studies need to be performed. At the present time, there are not factors that clearly indicate the response to anti-GER treatment in children with asthma. A recent study in school-age children with uncontrolled asthma showed that exhaled breath condensate $\mathrm{pH}$ measurement does not discriminate asymptomatic gastroesophageal reflux or the response to lansoprazole. ${ }^{23}$

The differentiation between acid and non-acidic reflux regarding respiratory symptoms may be important. Preliminary data suggest that non-acid reflux may result in respiratory symptoms. ${ }^{24}$ The possible role of non-acid reflux in worsening asthma control is unclear and larger, well-controlled studies are needed to validate this hypothesis.

The data showing no effect of acid suppression on asthma outcomes in children should not be generalised into treatment of GERD. The approved dose of PPIs may not have achieved adequate acid suppression in all 
children. A recent double-blind, placebo-controlled trial to investigate the effect of esomeprazole $40 \mathrm{mg}$ once or twice daily on asthma outcomes in adult patients with symptomatic GER revealed that both active doses were effective, but only esomeprazole $40 \mathrm{mg}$ twice daily demonstrated a significant improvement in forced expiratory volume in one second (FEV1). ${ }^{25}$ On the other hand, studies on PPIs using impedance measurements have failed to prove any effect of these drugs on nonacidic reflux. ${ }^{26}$ Supplemental therapies including pro-motility agents with effects apart from acid suppression have not been widely studied in children with asthma and GERD.

Surgical treatment may have a place in selected patients with GERD. Fundoplication is currently the anti-reflux procedure of choice in children with persistent or severe respiratory symptoms and reflux aspiration refractory to the medical treatment. In a recent prospective study in adults with GERD, both extra-oesophageal and oesophageal reflux symptoms decreased after treatment with esomeprazole and were reduced further after fundoplication. ${ }^{27}$ In a group of children with difficult-to-treat respiratory symptoms who did not respond to antireflux medical treatment, GERD was eliminated by anti-reflux surgery in almost all patients and respiratory symptoms improved in most. ${ }^{28}$

\section{Making rational decisions}

Physicians should conduct themselves with humility and responsibility in the decision-making process for patients with difficult-to-treat asthma and suspected GERD. Results do not actually support routine oesophageal pH testing to identify children who respond to PPIS, nor do they support indiscriminate trials of PPIS for poorly controlled asthma. Two specific questionnaires were found effective in distinguishing infants and young children with symptomatic GERD from healthy children. ${ }^{29}$ However, asthma is rare at this age and simple questions may help to identify children with symptomatic GER (see Table 1) ${ }^{30}$ If asymptomatic reflux is
Table 1: Questions for a symptom-based evaluation of gastroesophageal reflux in children

\section{Did you throw up?}

Did you feel like throwing up or feel nauseous?

Did you have a burning or painful feeling in the middle of your chest?

Did you have stomach ache above the belly button?

Did you have a sour taste, like the taste of throw-up, in the mouth?

Did it hurt to swallow food or drink?

suspected, treatment with PPIs is unlikely to ameliorate the symptoms of asthma; if symptomatic reflux is found, a three-month therapeutic trial with PPIs may be reasonable, but if there is no response, the drug should be immediately interrupted. There is a concerning increase in pneumonia ${ }^{31}$ and in activity-related bone fractures ${ }^{32}$ in children treated with PPIs that calls for a warning in the use of these medications. ${ }^{33}$

\section{Conclusions}

Many findings support a possible association between GERD and asthma in children. However, there is not enough evidence to support causality. Several gaps and areas of deficiency are still present, and they urgently require new studies, especially those with long-term follow-up.

When approaching a child with asthma and suspected GERD, physicians should balance their therapeutic decisions in between science and ethics. At one end, there is the principle that any correct treatment presupposes both the respect of epidemiological data and the knowledge of the pathophysiological mechanism(s) underlying the symptoms. At the other end, it should be never forgotten that treating the patient - in this case a child - and not the symptoms, remains the main principle at the heart of medicine. $\square$
1. Vandenplas $Y$, Rudolph $C D$, Di Lorenzo C, et al., Pediatric gastroesophageal reflux clinical practice guidelines: joint recommendations of the North American Society for Pediatric Gastroenterology, Hepatology, and Nutrition (NASPGHAN) and the European Society for Pediatric Gastroenterology, Hepatology, and Nutrition (ESPGHAN), J Pediatr Gastroenterol Nutr, 2009;49:498-547.

2. Field SK, Underwood $M$, Brant $R$, et al., Prevalence of gastroesophageal symptoms in asthma, Chest, 1996;109:316-22.

3. Hancox RJ, Poulton R, Taylor DR, et al., Associations between respiratory symptoms, lung function and gastrooesophagea reflux symptoms in a population-based birth cohort, Respir Res, 2006;7:142

4. Thakkar K, Boatright RO, Gilger MA, et al., Gastroesophageal reflux and asthma in children: a systematic review, Pediatrics, 2010;125:925-30.

5. Sherman PM, Hassall E, Fagundes-Neto U, et al., A global, evidence-based consensus on the definition of gastroesophageal reflux disease in the pediatric population Am J Gastroenterol, 2009;104:1278-95.

6. Blake K, Teague WG, Gastroesophageal reflux disease and childhood asthma, Curr Opin Pulm Med, 2013;19:24-9. El-Serag HB, Gilger M, Kuebeler M, et al., Extraesophageal associations of gastroesophageal reflux disease in children without neurologic defects, Gastroenterology, 2001,121.1294-9.

8. Andze $\mathrm{GO}$, Brandt ML, St Vil D, et al., Diagnosis and treatmen of gastroesophageal reflux in 500 children with respiratory
symptoms: the value of pH monitoring, $J$ Pediatr Surg, 1991;26:295-9.

9. Holbrook JT, Wise RA, Gold BD, et al., Lansoprazole for children with poorly controlled asthma: a randomized clinical trial IAMA, 2012;307:373-81.

10. Martin ME, Grunstein MM, Larsen GL, The relationship of gastroesophageal reflux to nocturnal wheezing in children with asthma, Ann Allergy, 1982:49:318-22

11. Gustafsson PM, Kjellman NI, Tibbling $L$, Bronchial asthma and acid reflux into the distal and proximal oesophagus, Arch Dis Child, 1990;65:1255-8.
12. Cinquetti M, Micelli S, Voltolina $C$, et al., The pattern of gastroesophageal reflux in asthmatic children, J Asthma, 2002;39:135-42.

13. Parsons JP, Mastronarde JG, Gastroesophageal reflux disease and asthma, Curr Opin Pulm Med, 2010; 16:60-3.

4. Harding SM, Richter JE, The role of gastroesophageal reflux in chronic cough and asthma, CHEST, 1997;111:1389-402.

15. de Benedictis FM, Carnielli V, de Benedictis D, Aspiration lung syndrome, Pediatr Clin North Am, 2009;56:173-90.

6. Asher MI, Montefort S, Bijorksten B, et al., Worldwide time trends in the prevalence of symptoms of asthma, allergic rhinoconjunctivitis, and eczema in childhood: ISAAC phases one and three repeat multicountry cross-sectional surveys, Lancet, 2006;368:733-43.

17. Dent J, El-Serag HB, Wallender MA, et al., Epidemiology of gastro-esophageal reflux disease: a systematic review, Gut, 2005;54:710-7.

18. de Benedictis FM, Bush A, Problematic severe asthma in children: the Pandora' box, Eur Respir Dis, 2011;7:91-6

9. Gibson PG, Henry RL, Coughlan JL, Gastro-oesophageal reflux treatment for asthma in adults and children, Cochrane Database Syst Rev, 2003;2:CD001496.

20. Kiljander TO, Harding SM, Field SK, et al., Effects of esomeprazole $40 \mathrm{mg}$ twice daily on asthma: a randomize placebo-controlled trial, Am J Respir Crit Care Med, 2006:173:1091-7.

21. Mastronarde JG, Anthonisen NR, Castro M, et al., Efficacy of esomeprazole for treatment of poorly controlled asthma N Eng/ J Med, 2009;360:1487-99.

, et al Acid suppression does not change respiratory symptoms in children with asthma and gastro-oesophageal reflux disease, Arch Dis 5; $90: 956-60$

23. Fitzpatrick AM, Holbrook JT, Wei CY, et al., Exhaled breath condensate $\mathrm{pH}$ does not discriminate asymptomatic gastroesophageal reflux or the response to lansoprazole treatment in children with poorly controlled asthma, J Allergy
24. Condino AA, Sondheimer J, Pan Z, et al., Evaluation of gastroesophageal reflux in pediatric patients with asthma using impedance-pH monitoring, J Pediat , 2006;149:216-9

25. Kiljander TO, Junghard O, Beckman O, Lind T, Effect of esomeprazole $40 \mathrm{mg}$ once or twice daily on asthma: a randomized, placebo-controlled study, Am J Respir Crit Care Med, 2010;181:1042-8.

26. Vela MF, Camacho-Lobato L, Srinivasan R, et al., Simultaneous intraesophageal impedance and pH measurement of acid and nonacid gastroesophageal reflux: effect of omeprazole Gastroenterology, 2001;120:1599-606.

27. Rantanen T, Kiljander T, Salminen P, et al. Reflux symptoms and side effects among patients with gastroesophageal reflux disease at baseline, during treatment with PPIS, and after Nissen fundoplication, World I Surg, 2013;37:1291-6

28. Mattioli G, Sacco O, Repetto P, et al., Necessity for surgery in children with gastrooesophageal reflux and supraoesophagea symptoms, Eur J Pediatr Surg, 2004;14:7-13.

9. Deal L, Gold BD, Gremse DA, et al., Age-specific questionnaires distinguish GERD symptom frequency and severity in infants and young children: development and initial validation,

J Pediatr Gastroenterol Nutr, 2005;41:178-85.

30. Nelson SP, Chen EH, Syniar GM, et al., Prevalence of symptoms of gastroesophageal reflux during childhood: a pediatric practice-based survey. Pediatric Practice Research Group Arch Pediatr Adolesc Med, 2000,154.150-4.

31. Lambert AA, Lam JO, Paik JJ, et al., Risk of communityacquired pneumonia with outpatient proton-pump inhibito therapy: a systematic review and meta-analysis, PLOS One

32. Lau AN, Tomizza M, Wong-Pack M, et al., The relationship between long-term proton pump inhibitor therapy and skeletal fralty, Endocrine, 2015:49:606-10.

3. Martinez FD, Children, asthma, and proton pump inhibitors. costs and perils of therapeutic creep, JAMA, 2012;307:406-7. 